\title{
Clinicopathologic Charateristics and Gallbladder Dysfunction in Patients with Endoscopic Bile Reflux
}

\author{
Youn Ju Na, Kyu Won Chung, Sun Young Yi \\ Department of Internal Medicine, Ewha Womans University School of Medicine, Seoul, Korea
}

\begin{abstract}
Objectives: To investigate clinicopathologic findings and gallbladder (GB) function in patients with endoscopic bile reflux at outpatients clinic.

Methods: We classified endoscopic bile reflux into two groups by bile reflux index (BRI). Those who scored above 14 were the BRI (+) group, and those below 14 were the BRI (-) group. We analyzed clinical characteristics, endoscopic findings including Helicobacter pylori, GB function by DISIDA scan, and electron microscope (EM) findings of endoscopic bile reflux. And we compared clinicopathologic characteristics and GB function between two groups. Results: Endoscopic bile reflux identified in $9.7 \%$ of all cases with gastrointestinal symptoms. There are cholecystectomy in $6.7 \%$, gastrectomy in $2.7 \%$, and GB dysfunction in $20.0 \%$. They had prominent gastrointestinal symptoms with variable endoscopic findings. Foveolar hyperplasia is the most common pathologic finding and $H$. pylori colonization of the stomach was inhibited in cases of bile reflux gastritis. Bile reflux also had distinguishable ultra-structural changes identifiable by EM. BRI (+) group had more old age, GB dysfunction than BRI (-) group. Clinical symptoms and endoscopic findings did not differ between the two groups of endoscopic bile reflux. Conclusion: Endoscopic bile reflux was common findings with young adults (30's) at outpatients clinic. Foveolar hyperplasia is common pathologic finding. GB dysfunction were identified as significant risk factors for BRI (+) group. (Ewha Med J 2013;36(1):18-25)
\end{abstract}

Key Words: Bile reflux; DISIDA; Electron microscopy; Gastritis; Helicobacter pylori

\section{Introduction}

Bile acid is very important for lipid digestion; however, it is known to be very toxic when in the stomach or esophagus. It has been associated with gastric or esophageal mucosal changes such as dysplasia, intestinal metaplasia, ulcers and malignancies. The toxicity of bile salts is dependent on the degree of hydrophobia [1].

Received: July 26, 2012, Accepted: September 28, 2012

Corresponding author: Sun Young Yi, Department of Internal Medicine, Ewha Womans University School of Medicine, 1071 Anyangcheon-ro, Yangcheon-gu, Seoul 158-710, Korea Tel: 82-2-2650-5575, Fax: 82-2-2655-2076 E-mail: syy@ewha.ac.kr
Deoxycholic acid, lithocholic acid, and hydrophobic bile salt cause cellular damage; however, hydrophilic bile salts such as ursodeoxycholic acid have no cytotoxic effects, even at high concentrations. The toxicity of hydrophobic bile salts has been shown in several cell types and tissues, including hepatocytes, erythrocytes, gastrointestinal mucosa and gallbladder (GB) mucosa [2-6].

Over the past years, endoscopic bile collection and bile stained mucosa of the stomach (endoscopic bile reflux) is a common finding in patients who have undergone gastric or biliary surgery [7-9] and was found to be predominant in females and the elderly [10]. Billous vomiting was recognized as an undesirable side effect in patients having gastric or biliary surgery and 
endoscopic bile reflux complained about various gastrointestinal symptoms. The pathogenesis of bile reflux gastritis was understood for postoperative bile vomiting following decreased gastric reserve function, but in un-operated stomachs, the pathogenesis of bile reflux gastritis was not known $[11,12]$. And there are very small studies of clinicopathologic characteristics of endoscopic bile reflux. Three methods used to diagnosis bile reflux gastritis are ambulatory esophageal $\mathrm{pH}$ monitoring, fiberoptic spectrophotometry (Bilitec, Medtronic Inc., Skovlunde, Denmark) [13], and the histological bile reflux index (BRI). The ambulatory $\mathrm{pH}$ monitoring and Bilitec methods are not readily available and are uncomfortable for the patients. Recently, instead of Billtec, the BRI was used for evaluation of bile reflux gastritis $[14,15]$. We used the BRI for evaluating grade of endoscopic bile reflux. We evaluated the clinicopathologic findings of endoscopic bile reflux and correlation between endoscopic bile reflux and GB dysfunction, Helicobacter pylori infection, and electron microscopy (EM) findings in relation to BRI.

\section{Methods}

\section{Patients}

From December 2006 to July 2007, 774 patients underwent gastroduodenoscopy in the outpatients clinic at Ewha Womans University Mokdong Hospital. We included endoscopic findings of bile collection or macroscopic bile stained mucosa in any site of the stomach. We also included patient history of gastrectomy and cholecystectomy. But we excluded the patients with antibiotics, corticosteroid, non-steroidal anti-inflammatory drugs within 2 weeks. We observed 75 cases of endoscopic bile reflux in a total of 774 endoscopy cases. Following patients' consent for a biopsy and questionnaire, we performed histological evaluation of 75 patients, DISIDA scan of 61, and EM study of 6 . Gastroduodenoendoscopy of all study were performed by one experienced endoscopist.

We evaluated personal characteristics such as age, body mass index, alcohol and coffee consumption, smoking, and history of surgery of 75 patients. Clinical symptoms such as bloating, nausea, epigastric pain, acid regurgitation, abdominal fullness, vomiting and cough were reviewed.

\section{Endoscopic and pathologic findings and gallbladder function}

We reported the presence of esophagitis, gastric erythema, atrophy, gastric metaplasia, ulcer, fold thickening, polyps and cancer. Two gastric biopsies were collected at antrum for measurement of BRI. The pathologic examination was performed by two experienced pathologist. The pathologic findings included foveolar hyperplasia, venous congestion, edema, acute inflammation, chronic inflammation, intestinal metaplasia and $H$. pylo$r i$ status. For evaluation of the GB, DISIDA scan was performed in 61 patients. GB contractility below $60 \%$ was defined as GB dysfunction [16].

\section{Bile reflux index}

The histological BRI measures the degree of edema in lamina propria, intestinal metaplasia (IM), chronic inflammation, and gastric $H$. pylori infection [17]. The BRI was originally derived by stepwise logistic regression analysis of the histological grades found in antral biopsies from 350 subjects in whom bile acid levels in gastric juice had been measured. Equation $[(7 \times \mathrm{Oed})+(3 \times \mathrm{IM})+(4 \times \mathrm{CI})-(6 \times H$. pylori $)]$ gave the best prediction of a raised gastric juice bile acid concentration. An index above 14 had a sensitivity of $70 \%$ and a specificity of $85 \%$ for a bile acid level over 1.0 $\mathrm{mmol} / \mathrm{L}$ (the upper limit of "physiological" reflux) [17]. We analyzed the BRI and assigned patients to 1 of 2 groups. Those with values above 14 were in the BRI (+) group, and those below 14 were in the BRI (-) group. In this system, endoscopic bile reflux was composed of bile reflux gastiritis (BRI (+) group; BRI values above 14) and endoscopic bile reflux without satisfying the index of bile reflux gastritis (BRI (-) group ; BRI values below 14).

\section{Electron microscopy}

Transmission EM findings in 6 patients with endoscopic bile reflux and 2 patients with normal finding were reviewed. Biopsied tissue was fixed in $1 \%$ osmium tetroxide, dehydrated, and embedded in epoxy resin. 
Approximately $1 \mu \mathrm{m}$ thick liver tissue sections were stained with toluidine blue and utra-thin sections (to $60 \sim 70 \mathrm{~nm}$ ) were cut with an ultramicrotome (Richert-Jung, Vienna, Austria) using a diamond knife. The thin sections were then stained with $1 \sim 2 \%$ aqueous uranyl acetate, followed by $1 \%$ lead citrate. Specimens were examined and photographed with a transmission electron microscope (H-7650, Hitachi co., Ibaraki-ken, Japan) at an accelerating voltage of $80 \mathrm{kV}$.

\section{Statistical analysis}

Data were analyzed using the SPSS ver. 13.0 (SPSS Inc., Chicago, IL, USA). Continuous variables are expressed as the mean $\pm \mathrm{SD}$, while categorical variables are presented as absolute values and percentages. Univariate analysis using either the $\mathrm{T}$ test for independent groups or chi-square test was performed between the BRI (+) group and the BRI (-) group. We compared groups with regards to clinical symptoms, endoscopic findings, histological examination and GB function by DISIDA scan. We evaluated risk factors for bile reflux gastritis among endoscopic bile reflux cases. For each variable, the odds ratio (OR) and 95\% confidence interval (CI) were reported. A two-tailed $\mathrm{P}<0.05$ was considered statistically significant.

\section{Results}

\section{Incidence and age distribution in patients with endoscopic bile reflux}

Among patients with gastrointestinal symptom, incidence of endoscopic bile reflux was $9.7 \%$. The mean age was $44 \pm 16$ years and the female to male ratio was 1.5:1. We found endoscopic bile reflux to be common in young adults with a female predominance in 20 and 30 year olds. Peak incidence of age is thirties (30's). There was an increase in the ratio of males to females in 70's (Fig. 1). The BRI (+) group included 25 patients, the BRI (-) group 50 patients. In the BRI (+) group, the mean age of patients was significantly increased compared to the BRI (-) group and the number of older age ( $>60$ years) was significantly different between the two groups $(\mathrm{P}=0.005)$.
2. Demographic features, symptoms and GB function in patients with endoscopic bile reflux

Mean BMI of the patients was $22.3 \pm 3.5 \mathrm{~kg} / \mathrm{m}^{2}$ with BMI over 25 in 23.5\%, history of alcohol in 36.0\%, coffee intake in $40.0 \%$, and smoking in $16.0 \%$. The history of surgery included cholecystectomy in $6.7 \%$, gastrectomy in $2.7 \%$, and other abdominal surgery in 26.7\%. The most common symptoms were abdominal fullness $(65.3 \%)$, nausea $(65.3 \%)$, epigastric pain $(64.0 \%)$, acid regurgitation (57.3\%), and bloating (56.0\%). In 61cases, DISIDA scan was performed. Patients with GB dysfunction (contractility below 60\%) represented $20.0 \%$ of all cases. No significant differences between the two groups were noted for obesity (BMI> $25 \mathrm{~kg} / \mathrm{m}^{2}$ ), alcohol history, coffee, smoking, prior abdominal surgery and clinical symptoms. The patients in the BRI (+) group had more GB dysfunction than those in the BRI (-) group $(\mathrm{P}=0.020)$ (Table 1). GB dysfunction are significant risk factors in patients for endoscopic bile reflux with adjusted age. The BRI (+) group correlated with GB dysfunction (OR, 2.41; 95\% CI, 0.62 to 9.31 ).

3. Endoscopic and pathologic findings in patients with endoscopic bile reflux

The common endoscopic findings were erythematous gastric mucosa (38.7\%), erosions (37.3\%), atrophic changes $(28.0 \%)$, reflux esophagitis $(7.4 \%)$ and ulcers

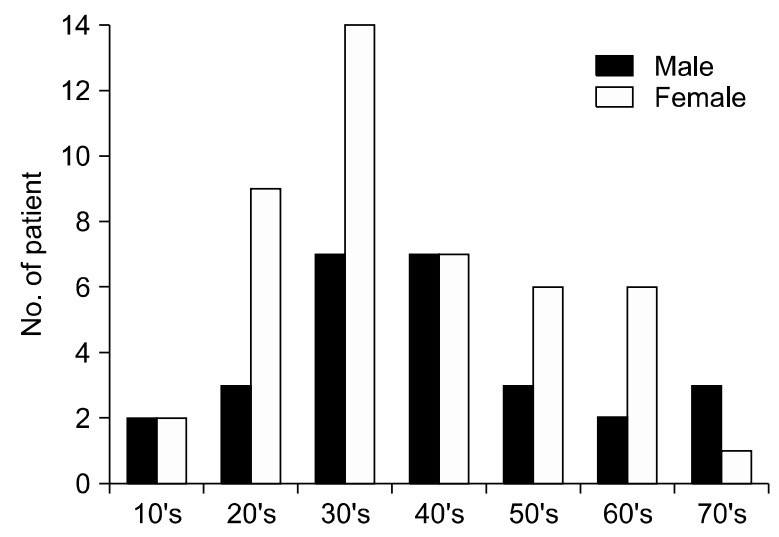

Fig. 1. Age and sex distribution of patients with endoscopic bile reflux. Peak incidence of age is thirties (30's). In 70's, there is an increase in the ratio of males to females. 
Table 1. Demographic features, symptoms and findings of DISIDA scan

\begin{tabular}{|c|c|c|c|c|}
\hline & Total patients $(n=75)$ & BRI $(+)(n=25)$ & BRI $(-)(n=50)$ & $P$ value \\
\hline Age (yr), mean $\pm S D$ (range) & $44 \pm 16(16 \sim 79)$ & 50.8 & 40.9 & 0.004 \\
\hline Age $>60$ yr $(n=15)$ & 20 & 40.0 & 10.0 & 0.005 \\
\hline $\mathrm{BMI}\left(\mathrm{kg} / \mathrm{m}^{2}\right)$, mean $\pm \mathrm{SD}$ & $22.3 \pm 3.5$ & 23.3 & 21.8 & 0.089 \\
\hline $\mathrm{BM} \mid>25$ & 23.5 & 32.0 & 20.0 & 0.251 \\
\hline Alcohol & 36.0 & 36.0 & 36.0 & 1.000 \\
\hline Coffee & 40.0 & 32.0 & 44.0 & 0.317 \\
\hline Smoking & 16.0 & 12.0 & 18.0 & 0.75 \\
\hline \multicolumn{5}{|l|}{ Surgery } \\
\hline No operation & 64.0 & 56.0 & 68.0 & 1.000 \\
\hline Cholecystectomy & 6.7 & 16.0 & 2.0 & \\
\hline Gastrectomy & 2.7 & 8.0 & 0.0 & \\
\hline Other abdominal surgery & 26.7 & 20.0 & 30.0 & \\
\hline \multicolumn{5}{|l|}{ Clinical symptoms } \\
\hline Bloating & 56.0 & 48.0 & 60.0 & 0.324 \\
\hline Nausea & 65.3 & 60.0 & 68.0 & 0.493 \\
\hline Epigastric pain & 64.0 & 64.0 & 64.0 & 1.000 \\
\hline Acid regurgitation & 57.3 & 52.0 & 60.0 & 0.509 \\
\hline Abdominal fullness & 65.3 & 52.0 & 72.0 & 0.086 \\
\hline Vomiting & 28.0 & 24.0 & 30.0 & 0.585 \\
\hline Cough & 26.7 & 16.0 & 32.0 & 0.140 \\
\hline Weight loss & 22.7 & 16.0 & 26.0 & 0.330 \\
\hline \multicolumn{5}{|l|}{ DISIDA scan $(n=61)$} \\
\hline GB function (contraction $\geq 60 \%$ ) & $61.3(n=46)$ & $55.6(n=10)$ & $83.7(n=36)$ & 0.020 \\
\hline GB dysfunction (contraction<60\%) & $20.0(n=15)$ & $44.4(n=8)$ & $16.3(n=7)$ & \\
\hline
\end{tabular}

Values are presented as percentage. BRI, bile reflux index; BMI, body mass index; GB, gall bladder.

Table 2. Endoscopic and pathologic findings

\begin{tabular}{|c|c|c|c|c|}
\hline Variable & Total $(n=75)$ & BRI $(+)(n=25)$ & BRI $(-)(n=50)$ & $P$ value \\
\hline \multicolumn{5}{|l|}{ Endoscopic findings } \\
\hline \multicolumn{5}{|l|}{ Esophagus } \\
\hline Normal & 44.4 & 52.0 & 42.0 & 0.232 \\
\hline Reflux esophagitis, minimal change & 48.1 & 48.0 & 52.0 & \\
\hline Reflux esophagitis, LA-A & 6.2 & 0.0 & 4.0 & \\
\hline Reflux esophagitis, LA-B & 1.2 & 0.0 & 4.0 & \\
\hline \multicolumn{5}{|l|}{ Stomach } \\
\hline Erythema & 38.7 & 48.0 & 34.0 & 0.241 \\
\hline Erosion & 37.3 & 44.0 & 34.0 & 0.399 \\
\hline Atrophic change & 28.0 & 40.0 & 22.0 & 0.102 \\
\hline Metaplastic change & 14.7 & 12.0 & 16.0 & 0.644 \\
\hline Ulcer & 8.0 & 12.0 & 6.0 & 0.367 \\
\hline Fold thickening & 1.3 & 0.0 & 2.0 & 0.477 \\
\hline Polyp & 4.0 & 4.0 & 4.0 & 1.000 \\
\hline \multicolumn{5}{|l|}{ Pathologic findings } \\
\hline Foveolar hyperplasia & 90.7 & 88.0 & 92.0 & 0.575 \\
\hline Venous congestion & 67.9 & 76.0 & 72.0 & 0.712 \\
\hline Edema & 66.7 & 100.0 & 58.0 & $0.000 *$ \\
\hline Acute inflammation & 43.2 & 48.0 & 46.0 & 0.870 \\
\hline Chronic inflammation & 70.4 & 84.0 & 72.0 & 0.251 \\
\hline Intestinal metaplasia & 19.2 & 28.0 & 16.0 & 0.221 \\
\hline Presence of Helicobacter pylori & 40.5 & 24.0 & 48.0 & $0.046 *$ \\
\hline
\end{tabular}

Values are presented as percentage. BRI, bile reflux index; LA-A, reflux esophagitis one or more mucosal breaks, no longer than $5 \mathrm{~mm}$, that do not extend between the tops of two mucosal folds; LA-B, reflux esophagitis one or more mucosal breaks, more than $5 \mathrm{~mm}$, that do not extend between the tops of two mucosal folds. $\star P<0.05$. 
(8.0\%). The endoscopic findings were not significantly different between the two groups. The histological alterations were foveolar hyperplasia (90.7\%), venous congestion (67.9\%), chronic inflammation (70.4\%), intestinal metaplasia (19.2\%), acute inflammation (43.2\%), H. pylori infection (40.5\%), and edema (66.7\%). In the BRI (+) group, edema was significantly higher than in the BRI (-) group (Table 2).

\section{H. pylori infection rate in patients with endoscopic bile reflux}

The incidence of $H$. pylori infection was significantly decreased in the BRI (+) group compared to the BRI

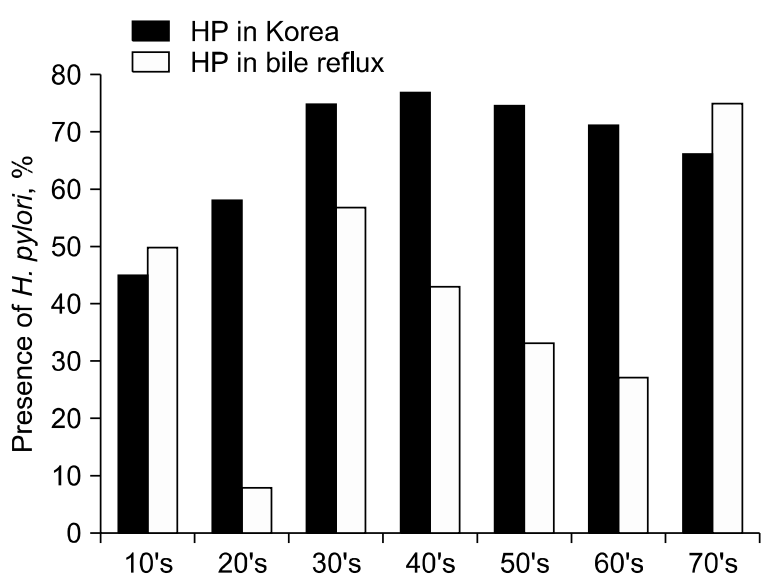

Fig. 2. The presence of Helicobacter pylori (HP) in patients with endoscopic bile reflux and the Korean population.
(-) group (24.0\% vs. $48.0 \%, \mathrm{P}<0.05)$ (Table 2$)$. Fig. 2 shows the presence of $H$. pylori in patients with endoscopic bile reflux compared to $H$. pylori in the Korean population in general. In teenagers and over 70 age groups, the incidence of $H$. pylori is higher in those with endoscopic bile reflux than in the general population, while the middle aged groups with endoscopic bile reflux have a significantly lower incidence of $H$. pylori than the Korean general population [18].

\section{EM findings in patients with endoscopic bile reflux}

Transmission EM shows findings of normal gastric mucosa and endoscopic bile reflux (Figs. 3, 4). The gastric mucosa of endoscopic bile reflux patients had decreased numbers, height, and mucus droplets of villi. The abnormality was aggravated as BRI count increased. Destruction of gastric mucus layers, dilated intercellular spaces were noted in cases of endoscopic bile reflux.

\section{Discussion}

Previously, we thought that gastrectomy or cholecystectomy induced upper gastrointestinal tract dysfunction, concentration of duodenal contents, and amount of food intake have been identified as possible important factors in the development of bile reflux gastritis $[7,8,19]$. Another possible risk factors were basal
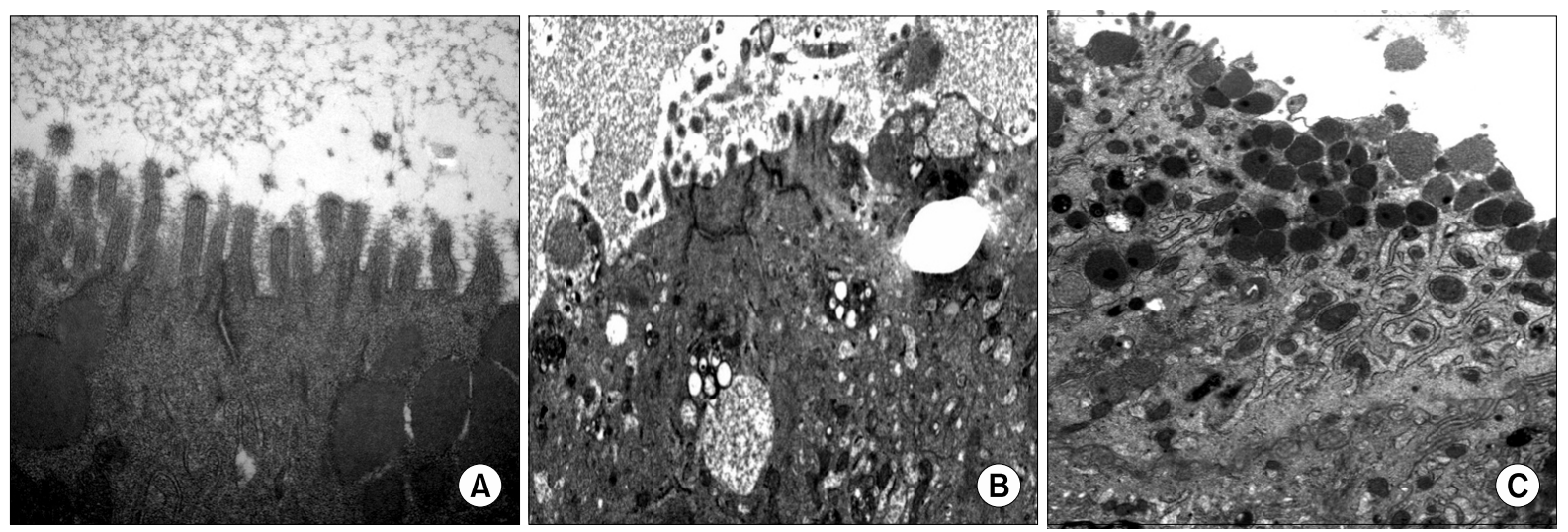

Fig. 3. Ultrastructure of gastric villi. (A) Ultrastructure of normal gastric mucosa. Number and height of villi were normal. (B) Ultrastructure of endoscopic bile reflux gastric mucosa (BRI<14). Numbers and height of villi are decreased. Also mucus droplets of villi are decreased. (C) Ultrastructure of endoscopic bile reflux of gastric mucosa (BRI>14). Numbers and height of villi are decreased. Also mucus droplets of villi are decreased $(\times 3,000)$. 

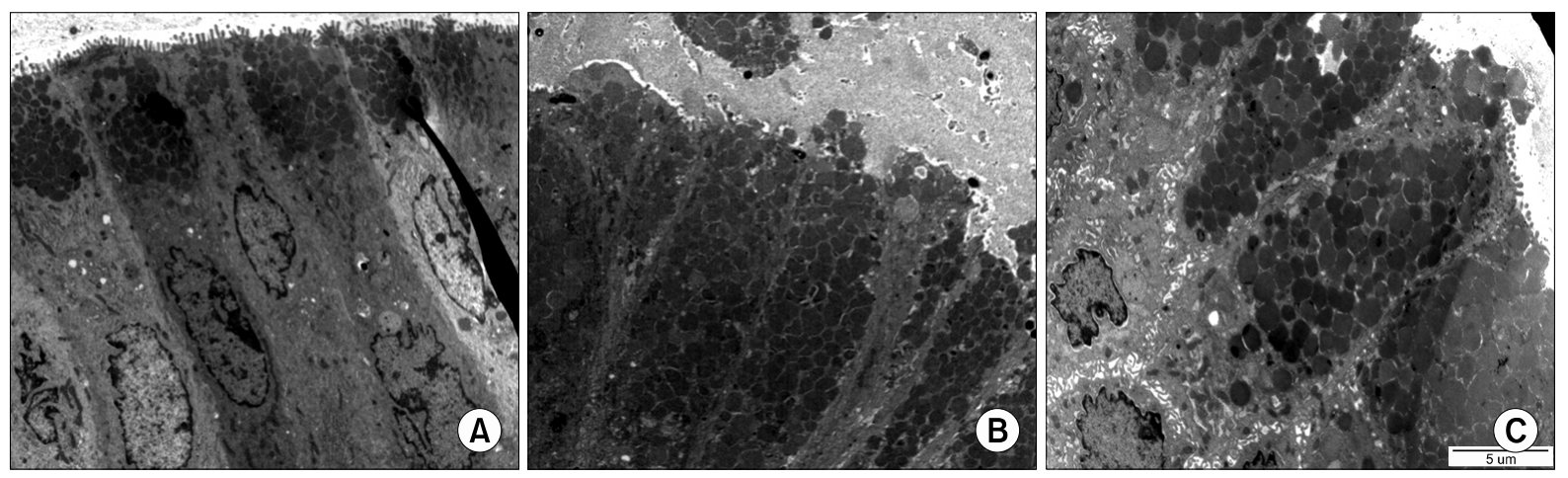

Fig. 4. Ultrastructure of gastric mucus layer and intracellular spaces. (A) Ultrastructure of normal gastric mucosa. There are no destruction of mucus layers and no dilated intercellular spaces. (B) Ultrastructure of endoscopic bile reflux gastric mucosa (BRI<14). There is slightly destruction of mucus layers. There are no dilated intercellular spaces. (C) Ultrastructure of endoscopic bile reflux gastric mucosa (BRI>14). There is slightly destruction of mucus layers and there are dilated intercellular spaces $(\times 5,000)$.

pyloric hypotension $[8,12,20-22]$, cholelithiasis $[7,8]$, pancreatitis, duodenal inflammation caused by adjacent inflammation (acute cholecystitis, pancreatitis) and drugs [9]. Also, sphincter of Oddi dysfunction or therapeutic biliary procedures without cholecystectomy are important factors related to bile reflux because this procedure disrupts the function of the sphincter of Oddi and also increases bile reflux [23]. Our results indicate an operation history involving the gastrectomy in only $2.7 \%$ and cholecystectomy in $6.7 \%$ of cases. In this study, we observed that $20 \%$ of endoscopic bile reflux cases had GB dysfunction. GB dysfunction may be a more significant cause than abdominal surgery. In the BRI (+) group, a significantly higher rate of GB dysfunction was found than in the BRI (-) group. GB dysfunction, sphincter malfunction, cholecystectomy and choledochoduodenostomy contribute to endoscopic bile reflux.

Irrespective of mechanism or grade of bile reflux gastritis, patients with endoscopic bile reflux has significant gastrointestinal symptoms. Between BRI (+) and BRI (-) groups, clinical symptoms were not different. Even the BRI (-) group had significant uncomfortable symptoms such as bloating, nausea, epigastric pain, acid regurgitation and abdominal fullness. The BRI (+) group had increased age, GB dysfunction, mucosal edema of the stomach, and a decrease in $H$. pylori positivity compared to the BRI (-) group. Some researchers observed no definite association between age and bile reflux $[9,24,25]$ while others reported a BRI (+) status to be more common in older than in younger individuals [26,27]. Our results identified age as a significant risk factor for bile reflux gastritis, possibly because bile was in contact with the gastric mucosa for a longer time, and older people were more likely to have pyloric dysfunction and a history of abdominal surgery such as gastric surgery and cholecystecomy [24].

Frequent endoscopic findings include erythema and erosion of gastric mucosa. Recent reports suggest that pathologic bile reflux was a risk factor of reflux esophagitis [10]. Our results showed reflux esophagitis in $7.4 \%$ and ulcers in $8.0 \%$. But there are minimal change reflux esophagitis in $48 \%$ of all case. Incidence of reflux esophagitis in BRI $(+)$ groups was higher than those of BRI (-) groups (not significant).

In one study, the histological feature most strongly associated with bile reflux was intestinal metaplasia, including all its subtypes. The prevalence of intestinal metaplasia was greatest in patients with both $H$. pylori infection and high bile acid concentrations [28,29]. $H$. pylori DNA may also be present in the bile when there are certain environmental changes, such as lowered pH. H. pylori does not colonize the bile duct epithelium $[17,30]$ and $H$. pylori was not significantly related to bile reflux in the stomach [31].

In our study, frequent pathologic findings in endoscopic bile reflux were foveolar hyperplasia, venous congestion, chronic inflammation and mucosal edema. 
In BRI (+) group, there were more cases of mucosal edema than intestinal metaplasia. Our results showed the BRI (-) group to have more $H$. pylori infection than the BRI $(+)$ group. It is possible that since bile reflux changes the $\mathrm{pH}$ of the stomach, $H$. pylori could not grow, limiting its presence in cases of reflux.

The experimental EM study showed that gastric acid induced mucosal dilated intercellular spaces in the esophagus [11,32] and proton pump inhibitors may repair esophageal mucosal integrity and induce dilated intercellular spaces. Also, bile acid exposure provoked disruption of the cell membrane [33] or dilated intercellular space of gastrointestinal tract mucosa [34,35]. Our EM results showed the BRI (+) group had more dilated intercellular spaces than the BRI (-) group and controls. Numbers and height of villi and mucus droplets in the BRI (+) group also were less than in the BRI $(-)$ and normal groups.

In conclusion, endoscopic bile reflux (9.7\%) is a very common disease encountered in the outpatients clinic. It was found to be common in young adults (30's) and more predominant in females. No history of prior abdominal surgery for all cases was 64\%. They had significant clinical symptoms and endoscopic findings such as reflux esophagitis (7.4\%) or ulcers $(8.0 \%)$. Bile reflux inhibited colonization of $H$. pylori in the stomach. Foveolar hyperplasia was the most common finding and distinguishable ultra-structural changes were identified by EM. BRI (+) group had more old age and GB dysfunction than BRI (-) group. But, BRI (-) group also had significant clinical symptoms and endoscopic finding such as reflux esophagitis.

\section{References}

1. Coleman R, Iqbal S, Godfrey PP, Billington D. Membranes and bile formation. Composition of several mammalian biles and their membrane-damaging properties. Biochem J 1979;178:201-208.

2. Moschetta A, vanBerge-Henegouwen GP, Portincasa P, Renooij WL, Groen AK, van Erpecum KJ. Hydrophilic bile salts enhance differential distribution of sphingomyelin and phosphatidylcholine between micellar and vesicular phases: potential implications for their effects in vivo. $J$ Hepatol 2001;34:492-499.
3. Moschetta A, Frederik PM, Portincasa P, vanBergeHenegouwen GP, van Erpecum KJ. Incorporation of cholesterol in sphingomyelin- phosphatidylcholine vesicles has profound effects on detergent-induced phase transitions. J Lipid Res 2002;43:1046-1053.

4. Velardi AL, Groen AK, Elferink RP, van der Meer R, Palasciano G, Tytgat GN. Cell type-dependent effect of phospholipid and cholesterol on bile salt cytotoxicity. Gastroenterology 1991;101:457-464.

5. Moschetta A, vanBerge-Henegouwen GP, Portincasa P, Palasciano G, Groen AK, van Erpecum KJ. Sphingomyelin exhibits greatly enhanced protection compared with egg yolk phosphatidylcholine against detergent bile salts. J Lipid Res 2000;41:916-924.

6. Bernstein H, Bernstein C, Payne CM, Dvorak K. Bile acids as endogenous etiologic agents in gastrointestinal cancer. World J Gastroenterol 2009;15:3329-3340.

7. Hoare AM, Jones EL, Alexander-Williams J, Hawkins CF. Symptomatic significance of gastric mucosal changes after surgery for peptic ulcer. Gut 1977;18:295-300.

8. Fountos A, Chrysos E, Tsiaoussis J, Karkavitsas N, Zoras OJ, Katsamouris A, et al. Duodenogastric reflux after biliary surgery: scintigraphic quantification and improvement with erythromycin. ANZ J Surg 2003;73: 400-403.

9. Abu Farsakh NA, Stietieh M, Abu Farsakh FA. The postcholecystectomy syndrome: a role for duodenogastric reflux. J Clin Gastroenterol 1996;22:197-201.

10. Vere CC, Cazacu S, Comănescu V, Mogoantă L, Rogoveanu I, Ciurea T. Endoscopical and histological features in bile reflux gastritis. Rom J Morphol Embryol 2005;46:269-274.

11. Taşkin V, Sedele M, Saka O, Kantarçeken B. The effect of duodenogastric reflux on Helicobacter pylori presence and gastric histopathologic changes. Turk $J$ Gastroenterol 2003;14:239-242.

12. Lujan-Mompean JA, Robles-Campos R, Parrilla-Paricio P, Liron-Ruiz R, Torralba-Martinez JA, Cifuentes-Tebar J. Duodenogastric reflux in patients with biliary lithiasis before and after cholecystectomy. Surg Gynecol Obstet 1993;176:116-118.

13. Bechi P, Cianchi F. Technical aspects and clinical indications of 24-hour intragastric bile monitoring. Hepatogastroenterology 1999;46:54-9.

14. Dixon MF, Mapstone NP, Neville PM, Moayyedi P, Axon AT. Bile reflux gastritis and intestinal metaplasia at the cardia. Gut 2002;51:351-355.

15. Sobala GM, King RF, Axon AT, Dixon MF. Reflux gastritis in the intact stomach. J Clin Pathol 1990;43: 303-306.

16. Kakhki VR, Zakavi SR, Davoudi Y. Normal values of 
gallbladder ejection fraction using 99mTc-sestamibi scintigraphy after a fatty meal formula. $J$ Gastrointestin Liver Dis 2007;16:157-161.

17. Sobala GM, O'Connor HJ, Dewar EP, King RF, Axon AT, Dixon MF. Bile reflux and intestinal metaplasia in gastric mucosa. J Clin Pathol 1993;46:235-240.

18. Kim JH, Kim HY, Kim NY, Kim SW, Kim JG, Kim JJ, et al. Seroepidemiological study of Helicobacter pylori infection in asymptomatic people in South Korea. $J$ Gastroenterol Hepatol 2001;16:969-975.

19. Koek GH, Vos R, Sifrim D, Cuomo R, Janssens J, Tack J. Mechanisms underlying duodeno-gastric reflux in man. Neurogastroenterol Motil 2005;17:191-199.

20. Shih WJ, Coupal JJ, Domstad PA, Ram MD, DeLand FH. Disorders of gallbladder function related to duodenogastric reflux in technetium-99m DISIDA hepatobiliary scintigraphy. Clin Nucl Med 1987;12:857-860.

21. Zhang ZH, Wu SD, Wang B, Su Y, Jin JZ, Kong J, et al. Sphincter of Oddi hypomotility and its relationship with duodenal-biliary reflux, plasma motilin and serum gastrin. World J Gastroenterol 2008;14:4077-4081.

22. Fein M, Bueter M, Sailer M, Fuchs KH. Effect of cholecystectomy on gastric and esophageal bile reflux in patients with upper gastrointestinal symptoms. Dig Dis Sci 2008;53:1186-1191.

23. Arroyo AJ, Burns JB, Huyghe WA, Dollman AE, Patel YP. Enterogastric reflux mimicking gallbladder disease: detection, quantitation and potential significance. $J$ Nucl Med Technol 1999;27:207-214.

24. Kuran S, Parlak E, Aydog G, Kacar S, Sasmaz N, Ozden $A$, et al. Bile reflux index after therapeutic biliary procedures. BMC Gastroenterol 2008;8:4.

25. Bordas JM, Elizalde I, Llach J, Mondelo F, Bataller $\mathrm{R}$, Teres J. Biliary reflux due to sphincter of Oddi ablation: a new pathogenetic explanation for long-term major biliary symptoms after endoscopic-sphincterotomy. Endoscopy 1996;28:642.

26. Bollschweiler E, Wolfgarten E, Putz B, Gutschow C, Holscher AH. Bile reflux into the stomach and the esophagus for volunteers older than 40 years. Digestion
2005;71:65-71.

27. Byrne JP, Romagnoli R, Bechi P, Attwood SE, Fuchs $\mathrm{KH}$, Collard JM. Duodenogastric reflux of bile in health: the normal range. Physiol Meas 1999;20:149-158.

28. Koek GH, Sifrim D, Lerut T, Janssens J, Tack J. Multivariate analysis of the association of acid and duodeno-gastro-oesophageal reflux exposure with the presence of oesophagitis, the severity of oesophagitis and Barrett's oesophagus. Gut 2008;57:1056-1064.

29. Kunsch S, Neesse A, Linhart T, Steinkamp M, Fensterer $\mathrm{H}$, Adler G, et al. Impact of pantoprazole on duodeno-gastro-esophageal reflux (DGER). Z Gastroenterol 2009;47:277-282.

30. Nakos A, Zezos P, Liratzopoulos N, Efraimidou E, Manolas K, Moschos J, et al. The significance of histological evidence of bile reflux gastropathy in patients with gastro-esophageal reflux disease. Med Sci Monit 2009;15:CR313-318.

31. Myung SJ, Kim MH, Shim KN, Kim YS, Kim EO, Kim HJ, et al. Detection of Helicobacter pylori DNA in human biliary tree and its association with hepatolithiasis. Dig Dis Sci 2000;45:1405-1412.

32. Stathopoulos P, Zundt B, Spelsberg FW, Kolligs L, Diebold J, Goke B, et al. Relation of gallbladder function and Helicobacter pylori infection to gastric mucosa inflammation in patients with symptomatic cholecystolithiasis. Digestion 2006;73:69-74.

33. Oumi M, Yamamoto T. A scanning electron microscope study on the effects of different bile salts on the epithelial lining of jejunal mucosa. Med Electron Microsc 2000;33: 11-15.

34. van Malenstein H, Farre R, Sifrim D. Esophageal dilated intercellular spaces (DIS) and nonerosive reflux disease. Am J Gastroenterol 2008;103:1021-1028.

35. Calabrese C, Fabbri A, Bortolotti M, Cenacchi G, Areni A, Scialpi C, et al. Dilated intercellular spaces as a marker of oesophageal damage: comparative results in gastro-oesophageal reflux disease with or without bile reflux. Aliment Pharmacol Ther 2003;18:525-532. 